\title{
Respuesta inmune humoral en vaquillonas vacunadas contra Tritrichomonas foetus y desafiadas con toros infectados
}

\author{
Riccio, M.B. ; Achilles, M.E. ${ }^{2}$; Cano, D. ; Lázaro, L. ${ }^{2}$; Cano, A. ${ }^{3}$; Campero, C.M.. ${ }^{3 *}$ \\ ${ }^{1}$ Becaria CONICET, ${ }^{2}$ Médicas veterinarias actividad privada, ${ }^{3}$ Grupo de Sanidad Animal, INTA Balcarce. \\ *Correspondencia: Patología Veterinaria, INTA Balcarce CC 276, (7620) Balcarce, Argentina. \\ Email: ccampero@balcarce.inta.gov.ar.
}

\begin{abstract}
Resumen
Riccio, M.B.; Achilles, M.E.; Cano, D.; Lázaro, L.; Cano, A.; Campero, C.M.: Respuesta inmune humoral en vaquillonas vacunadas contra Tritrichomonas foetus y desafiadas con toros infectados. Rev. vet. 19: 2, 101-108, 2008. Se evaluó el efecto de una vacuna oleosa a célula entera de Tritrichomonas foetus $\left(10^{8} /\right.$ dosis $)$ en vaquillonas desafiadas con toros infectados. Veintitrés vaquillonas fueron divididas en grupo $1(\mathrm{n}=11)$ control sin vacunar y grupo $2(n=12)$ inmunizadas en dos oportunidades por vía subcutánea. El grupo 2 se subdividió en 2 subgrupos de 6 animales cada uno, los cuales recibieron un tercer refuerzo vacunal por vías vaginal y vaginal e intranasal, respectivamente. Todas las vacunaciones se hicieron con 21 días de intervalo. A las 3 semanas de la última dosis, las vaquillonas fueron servidas durante 90 días con 2 toros infectados con T. foetus. Quincenalmente, hasta los 4 meses post servicio, se recolectó sangre para medir IgG y mucus cérvico vaginal (MCV) para cultivo y evaluación de $\operatorname{IgG}$ e IgA. La cuantificación de Ig se hizo por el test ELISA. La duración promedio de la infección para las vaquillonas vacunadas y controles fue de 44,9 días y 78,5 días, respectivamente $(\mathrm{p}<0,05)$. La parición fue de 8/12 $(66,6 \%)$ para los animales vacunados y $3 / 11(27,3 \%)$ para los controles $(p<0,05)$. Los valores ELISA séricos (VE) tuvieron un incremento luego de la segunda dosis vacunal $(\mathrm{p}<0,05)$ mientras que la IgA en el MCV fue significativamente superior en vacunados que en controles $(p<0,05)$, desde final del servicio hasta los 4 meses post servicio. No se observaron diferencias en los VE séricos ni en el MCV entre animales vacunados vías nasal y nasal e intravaginal. Se confirma la importancia de la estimulación vacunal sistémica y mucosal con antígenos de $T$. foetus con incrementos de la IgG sérica y de la IgA en el MCV acortando el período de infección y mejorando la eficiencia reproductiva en los animales vacunados. El método ELISA fue adecuado para la evaluación de la respuesta inmune en hembras infectadas y/o vacunadas.
\end{abstract}

Palabras clave: vaquillona, Tritrichomonas foetus, vacuna, inmunidad.

\begin{abstract}
Riccio, M.B.; Achilles, M.E.; Cano, D.; Lázaro, L.; Cano, A.; Campero, C.M.: Humoral immune response in vaccinated heifers against Tritrichomonas foetus and challenged with infected bulls. Rev. vet. 19: 2, 101-108, 2008. The performance of a whole-cell vaccine of Tritrichomonas foetus administered to heifers naturally challenged by mating with infected bulls, was determined. Twenty three heifers were divided into two groups: a control group $1(\mathrm{n}=11)$ without immunization, and group $2(\mathrm{n}=12)$ immunized two times subcutaneously with whole cells $\left(10^{8} /\right.$ dose) oil vaccine. Group 2 was divided in two groups of six heifers each, and received a third booster dose. One group was immunized by vaginal route and the other one by vaginal and intranasal routes, simultaneously. The females were vaccinated at 3 -week intervals. After 3 weeks of the last vaccine dose, heifers were mated with two bulls infected with $T$. foetus during 90 days. The mean duration of infection for vaccinated and control groups were 44.9 days and 78.5 days, respectively. Calving rates were 8/12 (66.6\%) for vaccinated heifers, and $3 / 11(27.3 \%)$ for control animals ( $<<0.05)$. ELISA test showed that serum immunoglobulin peaked before and during the breeding period. The vaccinated heifers developed an important response during the critical period of fetal loss, second and third month of the breeding time, and another month after the same period. ELISA method was sensitive and reliable for the evaluation of the systemic immune response in females infected and/or vaccinated with $T$. foetus.
\end{abstract}

Key words: heifer, Tritrichomonas foetus, vaccine, immunity. 


\section{INTRODUCCIÓN}

La trichomonosis bovina es una enfermedad de transmisión sexual ocasionada por el protozoo Tritrichomonas foetus que ocasiona pérdidas reproductivas en rodeos de diferentes países del mundo ${ }^{2,8}$. Si bien la enfermedad está controlada o erradicada en los países donde se utiliza la inseminación artificial, en aquellos con ganadería extensiva como el nuestro, la enfermedad todavía prevalece ${ }^{8}$. La inmunoprofilaxis contra $T$. foetus en hembras bovinas tiene como objetivo evitar las pérdidas reproductivas y sus secuelas ${ }^{2,9}$.

Diferentes investigaciones señalan que la pérdida fetal en hembras bovinas ocurre luego de 63 días post infección, aunque ya a los 50 días post infección es posible detectar lesiones placentarias y uterinas ${ }^{1,20}$. Se observó que las respuestas inmune e inflamatoria correlacionadas con la pérdida fetal, ocurren luego de 70 días post infección ${ }^{1,13,14}$. Es por ello esperable que los inmunógenos contra $T$. foetus en hembras bovinas generen una respuesta inmune capaz de eliminar el agente antes de que ocasione daño fetal (día 70-80 post infección), sin que necesariamente se evite la colonización temporaria del tracto genital ${ }^{3,9}$.

El uso de una vacuna efectiva contra la trichomonosis en hembras bovinas aumentaría la resistencia a la infección atenuando las pérdidas fetales y mejorando los programas de control 9,18. El objetivo del presente trabajo fue presentar la información generada por la respuesta humoral de vaquillonas vacunadas con antígenos de T. foetus y desafiadas mediante servicio natural con toros infectados, así como su repercusión en la eficiencia reproductiva.

\section{MATERIAL Y MÉTODOS}

Animales y vacunación. Se emplearon 23 vaquillonas vírgenes de 15-18 meses de edad, de raza Angus, Hereford y sus cruzas, mantenidas en condiciones de pastoreo extensivo en el INTA Balcarce. Las hembras provenían de un rodeo propio libre de brucelosis, tuberculosis, trichomonosis y campylobacteriosis. Las vaquillonas fueron seronegativas a la prueba de BPA para brucelosis y negativas a 2 muestreos de mucus cérvico vaginal (MCV) para diagnóstico de trichomonosis y campylobacteriosis mediante cultivo en caldo infusión hígado $(\mathrm{CIH})$ e inmunofluorescencia, respectivamente ${ }^{4,5}$.

La vacuna experimental se elaboró utilizando cultivos de célula entera de $T$. foetus en $\mathrm{CIH}$, con inactivante $\mathrm{y}$ adyuvante oleoso descriptos en un trabajo anterior ${ }^{12}$. Se utilizó la cepa B1 de T. foetus de referencia del INTA Balcarce, originalmente obtenida a partir de una infección natural de una vaca con piómetra ${ }^{7,12}$. Las hembras vacunadas fueron inmunizadas en tres oportunidades con intervalos de 21 días entre dosis. Los animales se dividieron al azar en grupos $1(\mathrm{n}=11$ controles sin vacunar) y $2(\mathrm{n}=12)$ vaquillonas vacunadas por vía subcutánea con $3,2 \times 10^{8}$ de $T$. foetus en un volumen de $6 \mathrm{ml}$, aplicados en la tabla del cuello en dos oportunidades. Luego de la segunda dosis, este último grupo fue subdivido al azar en 2 subgrupos de 6 animales cada uno, para recibir la tercera inmunización. El grupo 2a fue inmunizado por vía intravaginal (IV) con $0,5 \mathrm{ml}$ de una solución acuosa de $(\mathrm{HO})_{3} \mathrm{Al}$ conteniendo $0,74 \times 10^{8}$ de $T$. foetus aplicados intraluminal en el fondo de la vagina. El grupo $2 b$ fue vacunado con $0,5 \mathrm{ml}$ vía IV como el grupo 2a y $1 \mathrm{ml}$ de la suspensión oleosa aplicada por vía intranasal (IN) con $1,48 \times 10^{8}$ de $T$. foetus aplicada con pipeta plástica en caudal de la cavidad nasal.

Muestreos y cultivos. El MCV fue recolectado de la parte craneal de la vagina cercana al cérvix mediante pipeta de Cassou ${ }^{7}$ al inicio del ensayo, al momento de aplicar la tercera dosis vacunal y luego cada 15 días a partir de la $2^{a}$ semana de iniciado el servicio (día 85 post $1^{\circ}$ dosis vacunal) hasta 4 meses después de finalizado el mismo (día 256 post $1^{\circ}$ dosis vacunal). A partir de esta instancia se realizó un muestreo mensual hasta el día 284 post $1^{\circ}$ dosis vacunal. Se recolectaron además muestras de esmegma prepucial (EP) quincenalmente durante todo el servicio mediante pipeta de Cassou, el que fue sembrado en CIH para cultivo de T. foetus. Finalizado el servicio, se continuaron los muestreos prepuciales hasta un mes post-servicio, cuando los toros fueron vendidos. Las muestras de MCV y EP fueron sembradas in situ en $\mathrm{CIH}$, cultivadas en estufa a $37^{\circ} \mathrm{C}$ y examinadas microscópicamente durante 7 días ${ }^{7}$. En forma similar, una alícuota del MCV fue conservada a $-20^{\circ} \mathrm{C}$ para ser utilizada posteriormente para realizar test de ELISA. Con similar cronograma de muestreos, se obtuvo además sangre de la vena yugular cuyos sueros se mantuvieron a $-20^{\circ} \mathrm{C}$ hasta el momento de ser utilizados para detección de anticuerpos específicos contra T. foetus mediante test de ELISA.

Servicio y evaluación reproductiva. A los 21 días post última dosis vacunal, todas las vaquillonas fueron a servicio natural a campo con dos toros adultos, física y genitalmente aptos para el servicio e infectados artificialmente por vía intraprepucial con cultivos de la cepa de referencia B1 de T. foetus. A las tres semanas de la última dosis vacunal, todas las vaquillonas fueron desafiadas mediante servicio natural por 90 días con los 2 toros infectados artificialmente con $T$. foetus. El diagnóstico de preñez se efectuó en 2 ocasiones mediante ultrasonografía, la primera a los 80 días de iniciado el servicio y la segunda a los 18 días de finalizado el mismo. Se efectuaron además 4 tactos rectales seriados con el fin de confirmar el estado gestacional y detectar las pérdidas reproductivas. Se cuantificaron como pérdidas de preñez a aquellas hembras que habiendo sido diagnosticadas preñadas en exámenes previos, presentaban el útero no grávido o colecta amorfa en útero en los diferentes diagnósticos realizados. Las vaquillonas fueron evaluadas hasta el día 284 de iniciado el ensayo. 
Test ELISA para detectar anticuerpos contra T. foetus. Se determinaron los anticuerpos sistémicos contra $T$. foetus en suero sanguíneo y MCV mediante ELISA indirecto 2, 11, 13, 22 con algunas modificaciones. Las placas fueron sensibilizadas con $T$. foetus (cepa de referencia B1) suspendidos en buffer fosfato salino (PBS) ${ }^{12}$. Los MCV fueron homogeneizados, sonicados y procesadas por duplicado. En todos los casos se utilizaron sueros y MCV controles positivos y negativos, respectivamente, pertenecientes a animales hiperinmunizados contra $T$. foetus y bovinos sin contacto previo, utilizados en trabajos anteriores ${ }^{11,12}$. Para el caso de los sueros se utilizó IgG anti bovina conjugada con peroxidasa producida en conejo (Sigma, St. Louis, Mo, USA). La presencia de IgA en el MCV se evidenció con una anti-IgA bovina producida en conejo (Rabbit anti-bovine IgA Bethyl, Tx, USA) y una anti-IgG de conejo conjugada con peroxidasa (HRP-goat Anti-Rabbit IgG $(\mathrm{H}+\mathrm{L})$ Zymed $)^{10}$. Como cromógeno se utilizó ABTS pH 4,5 (Sigma, St. Louis, Mo, USA). Las placas fueron leídas mediante un lector de ELISA (Multiskan EX, Labsystems, Helsinki, Finland) a $405 \mathrm{~nm}$. Considerando las variaciones existentes en el procesado de las sucesivas placas, los valores de absorbancia expresados en densidad óptica fueron transformados a valores ELISA (VE) ${ }^{11,12}$.

Análisis estadístico. Se utilizó el límite de confianza del 95\% $(\mathrm{p}<0,05)$. Para los valores de ELISA, las absorbancias corregidas se compararon por contraste entre los grupos por el procedimiento del SAS ${ }^{12,21}$.

\section{RESULTADOS}

No se observaron reacciones generales adversas ni signos clínicos que afectaran el estado general de ninguno de los animales luego de la aplicación de las vacunas. Se observó en cambio, una ligera reacción local subcutánea en el lugar de la aplicación luego de 15 días de la $2^{\circ}$ dosis vacunal. Dicha reacción inflamatoria de tipo granulomatosa, de consistencia firme y con una reacción local de un diámetro promedio de $4 \mathrm{~cm}( \pm 1,5)$, se atribuyó al adyuvante oleoso y fue imperceptible luego de las tres semanas de su detección. No se observaron reacciones adversas en el área vaginal o en la mucosa nasal como secuela de la vacunación por dichas vías. La técnica de muestreo de MCV con pipeta de Cassou resultó un método satisfactorio, obteniéndose muestras abundantes y de buena calidad. El medio CIH resultó adecuado para el aislamiento de $T$. foetus siendo infrecuente la interferencia por contaminación. Los cultivos prepuciales realizados durante el servicio se mantuvieron positivos hasta el día 60 de inicio del mismo y luego se negativizaron hasta el final del ensayo.

En Figura 1 y Tabla 1 se detallan los resultados de los cultivos de MCV. El mayor porcentaje de infección de las hembras se observó durante el segundo mes de servicio (días 104 a 131 post $1^{\circ}$ dosis vacunal). En el grupo control se infectaron 10/11 hembras (90,9\%) mientras que en el grupo vacunado se infectaron $7 / 12$ hembras $(58,3 \%)(\mathrm{p}<0,05)$. La diferencia en los porcentajes de infección entre los grupos vacunados y control resultó significativa $(\mathrm{p}<0,05)$ para los días 104 y 160 post $1^{\circ}$ dosis vacunal. En los últimos 15 días de servicio (día 160 post $1^{\circ}$ dosis vacunal), 6/11 hembras del grupo control aún permanecían infectadas comparadas con $2 / 12$ de las hembras inmunizadas. A las 4 semanas post servicio (día 187 post $1^{\circ}$ dosis vacunal), 2/11 hembras controles seguían positivas en comparación con $1 / 12$ hembras vacunadas. Luego de 81 días de finalizado el servicio (día 242 post $1^{\circ}$ dosis vacunal), aún permanecía una hembra infectada en el grupo control.

También se observó que una vaquillona del grupo control $(1 / 11,9 \%)$ y tres del grupo vacunado $(3 / 12$, $25 \%$ ) resultaron negativas a los cultivos de MCV durante todo el ensayo, evidenciando ausencia de infección y permaneciendo preñadas hasta el final del ensayo. El tiempo promedio de infección en las hembras vacunadas fue menor que en las vaquillonas controles: 44,9 versus 78,5 días respectivamente, $\mathrm{p}<0,05$ (Tabla 1). El porcentaje de preñez final fue del 66,6\% (8/12) para los animales vacunados y $27,3 \%$ para los del grupo control $(3 / 11)(p<0,05$, Tabla 2). Dichos porcentajes de preñez se mantuvieron hasta finalizada la parición.

En el grupo vacunado, los VE séricos para $\operatorname{IgG}$ fueron más elevados a los 112 días post $1^{\circ}$ dosis vacunal (VE 90,3) y persistieron hasta la finalización del ensayo. En el grupo control se observaron valores de VE inferiores $(p<0,05)$ (Tabla 3 y Figura 2). A partir de la $2^{\circ}$ dosis vacunal (día 21 post $1^{\circ}$ dosis vacunal), se observaron importantes aumentos en los niveles de anticuerpos séricos en el grupo vacunado, mientras que la administración de la tercera dosis intramucosal produjo solo un leve aumento de los mismos (datos no presentados). Durante el período crítico de pérdidas reproductivas (60 días post desafío), se determinaron VE promedios más elevados en el grupo vacunado $(90,3)$ que en el grupo control $(35,2)$ (Tabla 3 ).

Finalmente, se registró una caída importante en los niveles de VE en los animales controles al día 112 post $1^{\circ}$ dosis vacunal mientras que en el grupo vacunado, este descenso fue más lento y tardío (día 200 post $1^{\circ}$ dosis vacunal) (Tabla 3 y Figura 2). Comparando el nivel de significancia del VE entre el grupo vacunado y el control, se establecieron diferencias significativas $(\mathrm{p}<0,05)$ a favor de los animales vacunados en los días $21,48,70$, $112,131,160$ y 200 post $1^{\circ}$ dosis vacunal, donde estaría incluido el período de servicio (Figura 2).

La inmunización por vía mucosal indujo una sostenida respuesta de IgA en el MCV. Al analizar estadísticamente los VE para IgA, los mismos fueron superiores en los grupos vacunados (IV/IN-IV) con respecto al grupo control $(\mathrm{p}<0,05)$ desde la finalización del servicio (VE 87,8 , día 160 post $1^{\circ}$ dosis vacunal) hasta el final del ensayo (VE 46,5, día 258 post $1^{\circ}$ dosis vacunal) (Tabla 3 y Figura 3). Al analizar la secuencia de cultivos, se observó que los aumentos de VE coincidieron con la negativización de las hembras (Figura 
Tabla 1. Secuencia de cultivos de mucus cérvico vaginal para $T$. foetus.

\begin{tabular}{|c|c|c|c|c|c|c|c|c|c|c|c|c|c|c|c|c|c|}
\hline \multirow[b]{2}{*}{$\operatorname{anim} \mathrm{N}^{\mathrm{o}}$} & \multirow[b]{2}{*}{ grupo } & \multicolumn{15}{|c|}{ días posteriores a $1^{\circ}$ dosis vacunal } & \multirow{2}{*}{$\begin{array}{c}\text { días } \\
\text { de } \\
\text { infección }\end{array}$} \\
\hline & & 0 & $85^{*}$ & $104 *$ & $112 *$ & $131 *$ & $145^{*}$ & $160^{*}$ & 173 & 187 & 200 & 214 & 228 & 242 & 256 & 284 & \\
\hline 3048 & $\mathrm{C}$ & - & - & + & + & + & + & + & - & - & - & - & - & - & - & - & 70,5 \\
\hline 3084 & $\mathrm{C}$ & - & + & + & + & + & + & - & - & - & - & - & - & - & - & - & 72 \\
\hline 3046 & $\mathrm{C}$ & - & - & + & + & + & + & + & - & + & + & + & - & - & - & - & 126 \\
\hline 3004 & $\mathrm{C}$ & - & - & - & - & - & - & - & - & - & - & - & - & - & - & - & 0 \\
\hline 3012 & $\mathrm{C}$ & - & + & + & + & - & + & + & - & - & - & - & - & - & - & - & 86 \\
\hline 3002 & $\mathrm{C}$ & - & + & + & + & + & - & + & + & - & - & - & - & - & - & - & 100 \\
\hline 3080 & $\mathrm{C}$ & - & - & + & + & + & - & - & - & - & - & - & - & - & - & - & 42.5 \\
\hline 3022 & $\mathrm{C}$ & - & - & + & + & + & - & - & - & - & - & - & - & - & - & - & 42,5 \\
\hline 3042 & $\mathrm{C}$ & - & + & + & + & + & - & + & - & - & - & - & - & - & - & - & 86 \\
\hline 3020 & $\mathrm{C}$ & - & - & + & + & + & + & - & + & + & - & + & + & + & - & - & 152,5 \\
\hline 3038 & $\mathrm{C}$ & - & + & + & + & + & - & + & - & - & - & - & - & - & - & - & 86 \\
\hline \multicolumn{2}{|c|}{$+/$ total } & $0 / 11$ & $5 / 11$ & $10 / 11$ & $10 / 11$ & $9 / 11$ & $5 / 11$ & $6 / 11$ & $2 / 11$ & $2 / 11$ & $1 / 11$ & $2 / 11$ & $1 / 11$ & $1 / 11$ & $0 / 11$ & $0 / 11$ & $\begin{array}{c}\text { promedio } \\
78,5\end{array}$ \\
\hline \multicolumn{2}{|c|}{$\%$ infectividad } & 0 & 45 & 90 & 90 & 81 & 45 & 54 & 18 & 18 & 9 & 18 & 9 & 9 & 0 & 0 & \\
\hline 3072 & V & - & + & + & + & - & - & - & - & - & - & - & - & - & - & - & 41,5 \\
\hline 3006 & V & - & - & - & - & - & - & - & - & - & - & - & - & - & - & - & 0 \\
\hline 3078 & $\mathrm{~V}$ & - & - & - & - & - & - & - & - & - & - & - & - & - & - & - & 0 \\
\hline 3018 & V & - & + & + & + & + & - & - & - & - & - & - & - & - & - & - & 58 \\
\hline 3036 & V & - & + & - & + & + & - & - & - & - & - & - & - & - & - & - & 58 \\
\hline 3010 & V & - & + & - & - & + & + & - & + & - & - & - & - & - & - & - & 100 \\
\hline 3030 & V & - & - & - & + & - & - & - & - & - & - & - & - & - & - & - & 24 \\
\hline 3074 & V & - & + & + & + & + & - & - & - & - & - & - & - & - & - & - & 58 \\
\hline 3054 & V & - & - & - & - & - & + & + & + & + & - & - & - & - & - & - & 56 \\
\hline 3086 & $\mathrm{~V}$ & - & - & - & - & - & - & - & - & - & - & - & - & - & - & - & 0 \\
\hline 3034 & $\mathrm{~V}$ & - & - & - & + & + & + & - & - & - & - & - & - & - & - & - & 44 \\
\hline 3060 & V & - & + & + & + & + & + & + & + & - & - & - & - & - & - & - & 100 \\
\hline \multicolumn{2}{|c|}{$+/$ total } & $0 / 12$ & $6 / 12$ & $4 / 12$ & $7 / 12$ & $6 / 12$ & $4 / 12$ & $2 / 12$ & $3 / 12$ & $1 / 12$ & $0 / 12$ & $0 / 12$ & $0 / 12$ & $0 / 12$ & $0 / 12$ & $0 / 12$ & $\begin{array}{c}\text { promedio } \\
44,9\end{array}$ \\
\hline \multicolumn{2}{|c|}{$\%$ infectividad } & 0 & 50 & 33 & 58 & 50 & 33 & 16 & 25 & 8 & 0 & 0 & 0 & 0 & 0 & 0 & \\
\hline
\end{tabular}

*servicio; grupo: C: control; V: vacunado

1), correlacionando significativamente $(\mathrm{p}<0,05)$ (día 160 post $1^{\circ}$ dosis vacunal) con un menor porcentaje de infección.

La aplicación de la tercera dosis intramucosal indujo una sostenida producción de IgA en el MCV durante el período del servicio y posterior al mismo (datos no presentados). Los VE de los grupos vacunados, IV versus IN-IV, no evidenciaron diferencias significativas

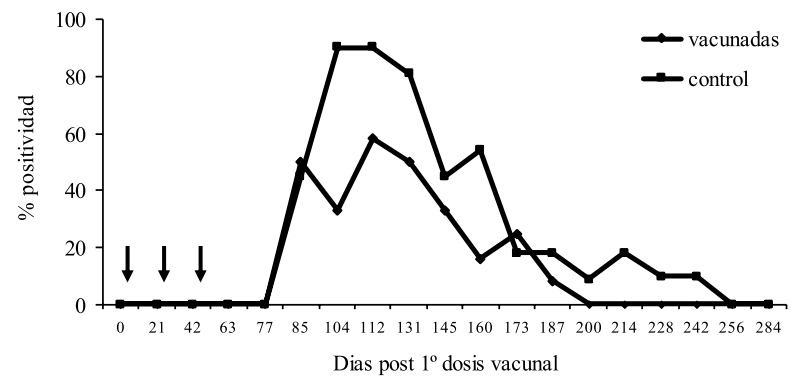

Figura 1. Porcentajes de cultivos positivos de T. foetus en mucus cérvico vaginal. Servicio: días 77 a 173. Flechas indican $1^{\circ}, 2^{\circ}$ y $3^{\circ}$ dosis vacunales.
( $>0,05)$ entre grupos (datos no presentados). Al analizar los VE de la IgG total en el MCV se observaron valores superiores $(p<0,05)$ en los grupos vacunados a partir de la segunda dosis post vacunal (día 21 post $1^{\circ}$ dosis vacunal) a lo largo de todo el trabajo con respecto a los controles (Tabla 3). Al comparar los VE entre los grupos vacunados, IV versus IN-IV, no se observaron diferencias significativas entre grupos $(p>0,05$, datos no presentados).

\section{DISCUSIÓN}

Los resultados abogan a favor de la eficacia de la vacuna inactivada a célula entera de $T$. foetus en la prevención de la infección genital, al inducir respuesta inmune efectiva sérica y genital con disminución de las pérdidas reproductivas con respecto a los animales controles sin vacunar. En forma similar y coincidiendo con otros autores, la vacuna utilizada no evitó la colonización del protozoo en el tracto reproductor femenino ${ }^{2}$, 15,19 . También se observaron algunos animales con resistencia natural a la infección donde no hubo colonización 
Tabla 2. Evolución de las gestaciones en los diferentes grupos.

\begin{tabular}{|c|c|c|c|c|c|c|c|}
\hline animal $N^{\circ}$ & grupo & $\begin{array}{c}1^{\circ} \\
\text { ecografía } \\
80 \text { días inicio } \\
\text { servicio }\end{array}$ & $\begin{array}{c}1^{\circ} \\
\text { tacto } \\
87 \text { días inicio } \\
\text { servicio }\end{array}$ & $\begin{array}{c}2^{\circ} \\
\text { ecografía } \\
\begin{array}{c}20 \text { días finalizado } \\
\text { servicio }\end{array}\end{array}$ & $\begin{array}{c}2^{\circ} \\
\text { tacto } \\
68 \text { días finalizado } \\
\text { servicio }\end{array}$ & $\begin{array}{c}3^{\circ} \\
\text { tacto } \\
84 \text { días finalizado } \\
\text { servicio }\end{array}$ & $\begin{array}{c}4^{\circ} \\
\text { tacto } \\
122 \begin{array}{c}\text { días finalizado } \\
\text { servicio }\end{array} \\
\end{array}$ \\
\hline 3048 & C & $\mathrm{P}$ & $\mathrm{P}$ & V & V & V & V \\
\hline 3084 & $\mathrm{C}$ & $\mathrm{P}$ & $\mathrm{P}$ & $\mathrm{P}$ & $\mathrm{P}$ & $\mathrm{P}$ & $\mathrm{P}$ \\
\hline 3046 & $\mathrm{C}$ & V & V & $\mathrm{P}$ & V & V & V \\
\hline 3004 & $\mathrm{C}$ & $\mathrm{P}$ & $\mathrm{P}$ & $\mathrm{P}$ & $\mathrm{P}$ & $\mathrm{P}$ & $\mathrm{P}$ \\
\hline 3012 & C & V & V & V & V & V & V \\
\hline 3002 & $\mathrm{C}$ & V & V & V & V & V & V \\
\hline 3080 & C & V & V & V & V & V & V \\
\hline 3022 & $\mathrm{C}$ & V & V & V & $\mathrm{P}$ & $\mathrm{P}$ & $\mathrm{P}$ \\
\hline 3042 & C & V & V & V & V & V & V \\
\hline 3020 & $\mathrm{C}$ & V & V & V & V & V & V \\
\hline 3038 & $\mathrm{C}$ & V & V & V & V & V & V \\
\hline $\mathrm{P} / \mathrm{V}$ & & $3 / 11$ & $3 / 11$ & $3 / 11$ & $3 / 11$ & $3 / 11$ & $3 / 11$ \\
\hline$\%$ preñez & & $27,3 \mathrm{a}$ & $27,3 \mathrm{a}$ & $27,3 \mathrm{a}$ & $27,3 \mathrm{a}$ & $27,3 \mathrm{a}$ & $27,3 \mathrm{a}$ \\
\hline 3072 & V & $\mathrm{P}$ & $\mathrm{P}$ & $\mathrm{P}$ & $\mathrm{P}$ & $\mathrm{P}$ & $\mathrm{P}$ \\
\hline 3006 & V & V & V & $\mathrm{P}$ & $\mathrm{P}$ & $\mathrm{P}$ & $\mathrm{P}$ \\
\hline 3078 & V & $\mathrm{P}$ & $P$ & $\mathrm{P}$ & $\mathrm{P}$ & $\mathrm{P}$ & $\mathrm{P}$ \\
\hline 3018 & V & V & V & $\mathrm{P}$ & $\mathrm{P}$ & $\mathrm{P}$ & $\mathrm{P}$ \\
\hline 3036 & V & V & V & V & V & V & V \\
\hline 3010 & V & V & V & V & V & V & V \\
\hline 3030 & V & $\mathrm{P}$ & $\mathrm{P}$ & $\mathrm{P}$ & $\mathrm{P}$ & $\mathrm{P}$ & $\mathrm{P}$ \\
\hline 3074 & V & V & V & $\mathrm{P}$ & $\mathrm{P}$ & $\mathrm{P}$ & $\mathrm{P}$ \\
\hline 3054 & V & V & V & V & V & V & V \\
\hline 3086 & V & $\mathrm{P}$ & $\mathrm{P}$ & $\mathrm{P}$ & $\mathrm{P}$ & $\mathrm{P}$ & $\mathrm{P}$ \\
\hline 3034 & V & V & V & V & $\mathrm{P}$ & $\mathrm{P}$ & $\mathrm{P}$ \\
\hline 3060 & V & V & V & V & V & V & V \\
\hline $\mathrm{P} / \mathrm{V}$ & & $4 / 12$ & $4 / 12$ & $7 / 12$ & $8 / 12$ & $8 / 12$ & $8 / 12$ \\
\hline \% preñez & & $33 a$ & $33 a$ & $58 \mathrm{~b}$ & $66,6 \mathrm{~b}$ & $66,6 \mathrm{~b}$ & $66,6 \mathrm{~b}$ \\
\hline
\end{tabular}

Letras distintas al comparar los porcentajes de preñez entre animales controles y vacunados indican diferencias significati$\operatorname{vas}(\mathrm{p}<0,05)$.

genital de $T$. foetus ( $9 \%$ y $25 \%$ en los grupos control y vacunado, respectivamente), en coincidencia con otros autores ${ }^{3}$. Se constató la presencia de una hembra del grupo control que permaneció infectada hasta 12 semanas post finalización del servicio, confirmando lo mencionado por otros autores que detectaron hembras infectadas hasta 12 a 25 semanas post desafío ${ }^{15,16,19,22}$.

En nuestro ensayo, el porcentaje de hembras control infectadas a las 4 semanas post servicio fue menor $(18 \%)$ comparada con otros trabajos ${ }^{15,19}$. Es factible que varias hembras se hayan liberado de la infección en fechas cercanas a la finalización del servicio, presentando celo luego de retirado el toro.

El tiempo promedio de persistencia de la infección en las hembras de ambos grupos difirió significativamente coincidiendo con otros trabajos que utilizaron el desafío con toro infectado ${ }^{19}$. Aquí, el tiempo promedio de infección por T. foetus en hembras vacunadas (7 se- manas) fue mayor que en trabajos previos donde la infección genital persistió un promedio de 3 a 4 semanas ${ }^{7}$, $15,17,19$, pudiendo llegar a 6 o 7 semanas ${ }^{2,16}$. Sin embargo, considerando el período crítico de 9 a 17 semanas post infección con $T$. foetus donde suceden la mayoría de las pérdidas fetales ${ }^{1,20}$, la liberación de la infección en las hembras vacunadas en nuestro ensayo habría ocurrido previamente a dicho período. Otros autores, empleando una vacuna a célula entera de $T$. foetus, tuvieron preñeces de 66,6 a $55,5 \%$ para el grupo vacunado y de 33,3 a $13,3 \%$ para el grupo no vacunado ${ }^{17}$, resultados aproximados a los registrados en este trabajo.

La respuesta inmune sistémica estuvo condicionada al tipo de inmunógeno empleado y a las diferentes vías de inmunización. En las hembras vacunadas se determinó un importante incremento de anticuerpos séricos post $2^{\circ}$ dosis vacunal sistémica, pero no se registraron dichos aumentos luego de la 
Tabla 3. Comparación de los valores ELISA en suero ( $\operatorname{IgG}$ ) y mucus cérvico vaginal ( $\operatorname{IgG}$, IgA) en animales vacunados y controles.

\begin{tabular}{lcccccccccc}
\hline & & \multicolumn{8}{c}{ días post primera dosis vacunal } \\
tipo de Ig y fluido & grupo & 0 & 21 & 48 & $70^{*}$ & $112^{*}$ & $131^{*}$ & $160^{*}$ & 200 & 258 \\
\hline \multirow{2}{*}{ IgG en suero } & vacunado & $7,6 \mathrm{a}$ & $19,7 \mathrm{a}$ & $70,1 \mathrm{a}$ & $74,4 \mathrm{a}$ & $90,3 \mathrm{a}$ & $84,0 \mathrm{a}$ & $51,8 \mathrm{a}$ & $47 \mathrm{a}$ & $12,5 \mathrm{a}$ \\
& control & $6,1 \mathrm{a}$ & $-5,3 \mathrm{~b}$ & $22,4 \mathrm{~b}$ & $35,3 \mathrm{~b}$ & $35,2 \mathrm{~b}$ & $-0,5 \mathrm{~b}$ & $2,3 \mathrm{~b}$ & $8,6 \mathrm{~b}$ & $-2,8 \mathrm{a}$ \\
& & & & & & & & & & \\
\multirow{2}{*}{ IgG en MCV } & vacunado & $1,3 \mathrm{a}$ & $10,3 \mathrm{a}$ & $23,3 \mathrm{a}$ & $28 \mathrm{a}$ & $28,9 \mathrm{a}$ & $35,6 \mathrm{a}$ & $46 \mathrm{a}$ & $50,1 \mathrm{a}$ & $49 \mathrm{a}$ \\
& control & $1,8 \mathrm{a}$ & $4,9 \mathrm{a}$ & $10,5 \mathrm{~b}$ & $9,7 \mathrm{~b}$ & $10,2 \mathrm{~b}$ & $21 \mathrm{~b}$ & $35,2 \mathrm{~b}$ & $40,5 \mathrm{~b}$ & $38,5 \mathrm{~b}$ \\
\multirow{2}{*}{ IgA en MCV } & vacunado & $-1,1 \mathrm{a}$ & $1,3 \mathrm{a}$ & $2,4 \mathrm{a}$ & $5,8 \mathrm{a}$ & $10,8 \mathrm{a}$ & $23,4 \mathrm{a}$ & $87,8 \mathrm{a}$ & $84,7 \mathrm{a}$ & $46,5 \mathrm{a}$ \\
& control & $1,9 \mathrm{a}$ & $7,9 \mathrm{a}$ & $6,8 \mathrm{a}$ & $0,9 \mathrm{a}$ & $3,0 \mathrm{a}$ & $28,7 \mathrm{a}$ & $51,4 \mathrm{~b}$ & $41,7 \mathrm{~b}$ & $27,4 \mathrm{~b}$ \\
\hline
\end{tabular}

*Servicio. Letras diferentes entre columnas poseen diferencias significativas $(\mathrm{p}<0,05)$ aplicadas en cada Ig y para cada fluido analizado.

$3^{\circ}$ dosis aplicada por vía intramucosal. Además, se determinó el mayor nivel sérico durante el primer mes de servicio en el grupo vacunado. Similar respuesta inmune, entre 3 y 8 semanas post $2^{\circ}$ dosis vacunal, fue descrita al evaluar el mismo tipo de vacuna ${ }^{15}$.

En las hembras inmunizadas, la respuesta inmune sistémica fue de mayor duración y más efectiva en la liberación de la enfermedad que en las hembras del grupo control. Las vaquillonas inmunizadas presentaron menor persistencia promedio de la infección y mayores índices de preñez. Además, para el grupo vacunado, el valor medio de ELISA fue mayor durante el servicio, estableciendo en algunos casos (21, 48, 70, 112, 131, 160 y 200 días post $1^{\circ}$ dosis vacunal) diferencias significativas con el grupo control.

La presencia de anticuerpos séricos en el grupo control, previo y durante el servicio, coincide con trabajos que señalan la presencia de anticuerpos a $T$. foetus en el suero de animales sanos ${ }^{6,22}$. El aumento de los anticuerpos séricos en el grupo control durante el servicio respondería a la estimulación sistémica

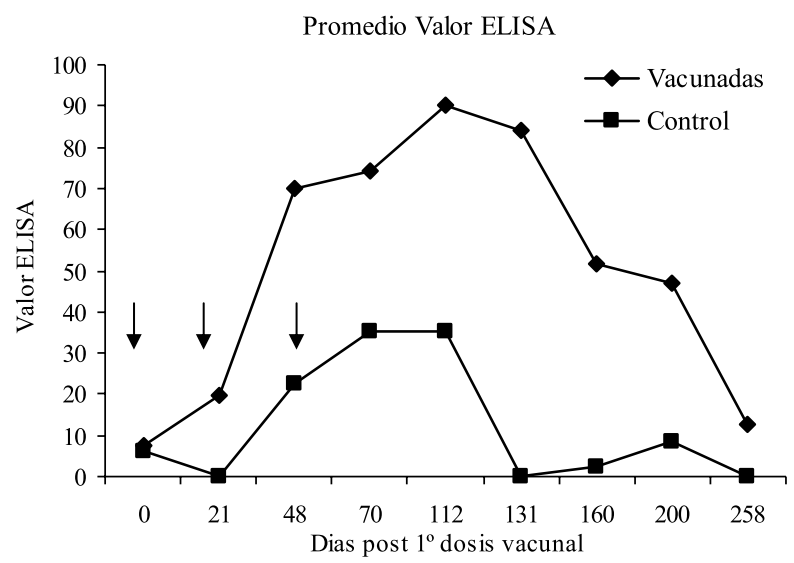

Figura 2. Promedios de IgG sérica a T. foetus expresada en VE en los grupos control y vacunado. VE entre 21 y 200 días fueron significativamente distintos entre grupos $(p<0,05)$. Flechas indican $1^{\circ}, 2^{\circ}$ y $3^{\circ}$ dosis vacunales. que se produce en infecciones naturales con incrementos de $\mathrm{IgG}_{1}{ }^{6,22}$. Trabajos previos reportan que un refuerzo vacunal ("booster") vía sistémica produce, luego de 2 a 3 semanas, un rápido aumento de $\mathrm{IgG}$ sérica que estimula su transporte hacia las secreciones vaginales ${ }^{13,15}$.

La aplicación de dosis vacunal parenteral en adyuvante oleoso, como las realizadas en la presente experiencia, permite suponer que los aumentos séricos observados corresponden a IgG. Además, debido al adyuvante y vías utilizadas, se podría especular en un incremento en los niveles genitales de IgA. La aplicación de un refuerzo vacunal en la mucosa genital implica un importante aumento de IgA local ${ }^{13}$, resultando esta inmunización en el mismo sitio de infección una importante opción vacunal. Finalmente, al considerar el período crítico de pérdidas fetales, luego de 60 días post desafío (131 a 160 días post $1^{\circ}$ dosis vacunal), donde se requiere una sólida respuesta inmune que evite el efecto patógeno de $T$. foetus, los valores de ELISA séricos aquí registrados fueron mayores en el grupo vacunado. En los animales vacunados la infección ge-

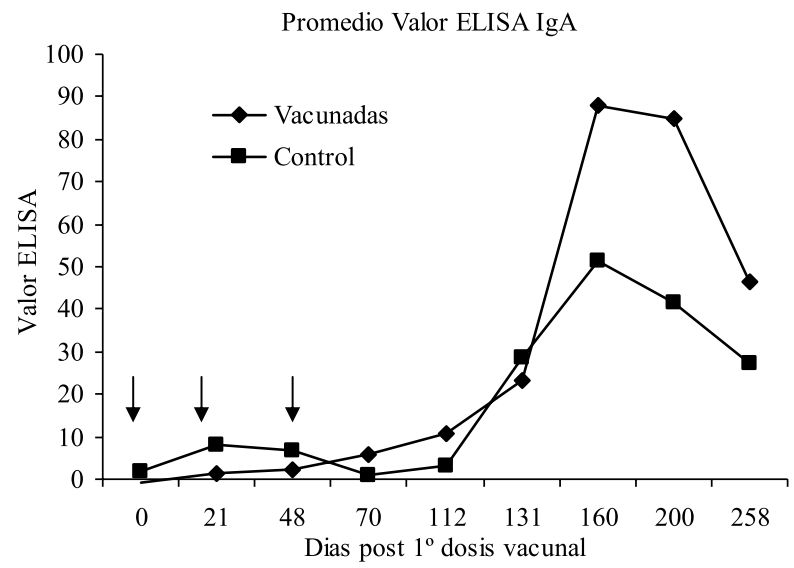

Figura 3. Valores de IgA anti-T. foetus en mucus cérvico vaginal de vaquillonas vacunadas y controles. VE entre 160 y 258 días fueron significativamente distintos entre grupos $(\mathrm{p}<0,05)$. Flechas indican $1^{\circ}, 2^{\circ}$ y $3^{\circ}$ dosis vacunales. 
nital decreció del 90,3\% a los 41 días de comenzado el servicio (112 post $1^{\circ}$ dosis vacunal) al $51,8 \%$ al final del servicio (día 160 post $1^{\circ}$ dosis vacunal). Durante ese lapso de 47 días la infección genital coexistió con altos niveles de anticuerpos séricos. Coincidentemente con la liberación de la infección, hacia el final del período de servicio, los niveles séricos disminuyeron en forma considerable.

Los elevados niveles de IgA en el MCV se correlacionaron positivamente con cultivos negativos a $T$. foetus avalando la efectividad de IgA para promover la liberación genital del protozoario. Además, a pesar que la respuesta de IgA genital inducida por vacunas coincidió en el tiempo con la respuesta natural en hembras sin inmunizar, la respuesta inducida en los animales vacunados fue significativamente mayor (VE 87,8) con respecto a los animales controles (VE 51,4). El aumento de la magnitud de la respuesta genital de $\operatorname{IgA}$ fue producto del estímulo local inducido por la vacuna y del efecto adicional del desafío generado por los toros infectados con $T$. foetus durante el servicio, evidenciándose por la respuesta local en las hembras controles. La presencia de $T$. foetus en el tracto genital estimuló una respuesta de IgA hacia final del servicio y luego del mismo.

Los VE para IgG en el MCV de los animales vacunados fueron significativamente superiores $(p<0,05)$ con respecto a los animales controles a lo largo de todo el ensayo. El aumento de los VE observados luego de última dosis vacunal aplicada por vía parenteral permite sugerir que la IgG sistémica difundió desde el lumen vascular hacia la secreción genital. Además se observó que los aumentos de IgG en MCV no fueron influenciados por la administración de una tercera dosis aplicada vía mucosal, lo que indica la importancia de la vía de inoculación de los inmunógenos.

En conclusión, surge que la estimulación vacunal sistémica y mucosal con antígenos de $T$. foetus generó incrementos de $\operatorname{IgG}$ en suero y de IgA en MCV, acortando el período de infección y mejorando la eficiencia reproductiva en los animales vacunados. El método ELISA utilizado en el presente ensayo resultó de utilidad para la detección de la respuesta inmune de $T$. foetus.

\section{REFERENCIAS}

1. Anderson M, BonDurant R, Corbeil R, Corbeil L. 1996. Inmune and inflammatory responses to reproductive tract infection with Tritrichomas foetus in immunized and control heifers. J Parasitology 82: 595-600.

2. BonDurant RH, Corbeil RR, Corbeil LB. 1993. Immunization of virgin cows with surface antigen TF1.17 of Tritrichomonas foetus. Infect Immun 61: 1385-1394.

3. BonDurant RH. 1997. Pathogenesis, diagnosis and management of trichomoniasis in cattle. Vet Clin N Am Food Anim Pract 13: 345-361.
4. Campero CM, Catena M, Cano D. 1986. Caldo infusión hígado para el cultivo de Tritrichomonas foetus. Vet Arg 3 : $80-81$.

5. Campero CM, Ballabene N, Cipolla A, Zamora A. 1987. Dual infection in bulls with campylobacteriosis and trichomoniasis: treatment with dimetridazole chlorhydrate. Aust Vet J 64: 320-321.

6. Campero CM, Cano D, Rossetti O, Marcovecchio F, Cosentino B, Marcone J, Carracino M. 1998. Vacunación subcutánea e intravaginal contra tricomoniasis en vaquillonas. Rev Med Vet 79: 347-352.

7. Campero CM, Rossetti O, Cano D, Bretschneider G, Roppel M. 1999. Inmunización en vaquillonas mediante vacuna de membrana de Tritrichomona foetus. Vet Arg 154: 250-262.

8. Campero CM, Cobo ER. 2006. Tritrichomonas foetus: patogénesis de la mortalidad embrionaria/fetal, caracterización de antígenos vacunales y respuesta inmune inducida. Rev Med Vet 87: 47-56.

9. Campero CM, Gottstein B. 2007. Tritrichomonosis. Control measures. In: Protozoal abortion in farm ruminants: guidelines for diagnosis and control (Ortega-Mora L, Gottstein B, Conraths FJ, Buxton D Ed), CABI Pub, Wallingford, p. 290-301.

10. Cobo ER, Cano D, Campero CM. 2001. Experimental infection with Tritrichomonas suis in heifers. Vet Parasitol 99: 73-78.

11. Cobo ER, Cano D, Rossetti O, Campero CM. 2002. Heifers immunized with whole-cell and membrane vaccines against Tritrichomonas foetus and naturally challenged with an infected bull. Vet Parasitol 109: 169-184.

12. Cobo ER, Morsella C, Cano D, Cipolla A, Campero CM. 2004. Immunization in heifers with dual vaccines containing Tritrichomonas foetus and Campylobacter fetus antigens using systemic and mucosal routes. Theriogenology 62: 1367-1382.

13. Corbeil LB, Anderson ML, Corbeil RR, Eddow JM, BonDurant RH. 1998. Female reproductive tract immunity in bovine trichomoniasis. Am J Reprod Immunol 39: 189-198.

14. Corbeil LB, Campero CM, Rhyan JC, BonDurant RH. 2003. Vaccines against sexually transmitted disease. Repr Biol Endocrin 118: 1-6.

15. Gault RA, Kvasnicka WG, Hanks D, Hanks M, Hall MR. 1995. Specific antibodies in serum and vaginal mucus of heifers inoculated with a vaccine containing Tritrichomonas foetus. Am J Vet Res 56: 454-459.

16. Herr S, Ribeiro LM, Claassen E, Myburgh JG. 1991. A reduction in the duration of infection with Tritrichomonas foetus following vaccination in heifers and the failure to demonstrate a curative effect in infected bulls. Onderstepoort J Vet Res 58: 41-45.

17. Hudson D, Ball L, Cheney J, Mortimer R, Bowen B, Marsh D, Peetz, R. 1993. Testing of trichomoniasis vaccine in heifers mated to infected bulls. Theriogenology 39 : 937-943.

18. Kvasnicka WG, Taylor RE, Huang JC, Hanks D, Tronstad RJ, Bosomworth A, Hall MR. 1989. Investigations of the incidence of bovine trichomoniasis in Nevada and 
of the efficacy of immunizing cattle with vaccines containing Tritrichomonas foetus. Theriogenology 31: 963-971.

19. Kvasnicka WG, Hanks D, Huang JC, Hall MR, SandBlind D, Chavez L, Acree WM. 1992. Clinical evaluation of the efficacy of inoculating cattle with a vaccine containing Tritrichomonas foetus. Am J Vet Res 53: 2023-2027.

20. Parsonson IM, Clark BL, Dufty JH. 1976. Early pathogenesis and pathology of Tritrichomonas foetus infection in virgin heifers. J Comp Pathol 86: 59-66.
21. SAS Institute Inc. 1987. SAS/STAT. Guide for personal computers. Version 6 Ed, Cary, NC, p. 320-392.

22. Skirrow SZ, Bondurant RH. 1990. Immunoglobulin isotype of specific antibodies in reproductive tract secretions and sera in Tritrichomonas foetus-infected heifers. Am J Vet Res 51: 645-653.

\section{Asociación Cooperadora de la Facultad de Ciencias Veterinarias}

Personería Jurídica No 647/92 y 912/00

Sargento Cabral 2139

3400 Corrientes

La Asociación Cooperadora de la Facultad de Ciencias Veterinarias fue constituida el 10 de diciembre del año 1991 como entidad de bien público, con el objeto de promover y coadyuvar las actividades científicas, educativas y culturales relacionadas con las Ciencias Veterinarias. En tal sentido, implementa acciones para colaborar con la enseñanza, extensión, actualización y difusión científica que realiza dicha Casa de Estudios.

\section{Beneficios que brinda a sus asociados:}

- Fotocopias con descuentos especiales del 20\% en la Fotocopiadora COPIAS.COM que funciona dentro del predio de la Facultad de Ciencias Veterinarias.

- $10 \%$ de descuento para la adquisición de libros de la Editorial Inter-Médica.

- $10 \%$ de descuento en las compras de medicamentos e insumos para trabajos prácticos hospitalarios. 